\title{
Erratum: Early kinetic decoupling of dark matter: When the standard way of calculating the thermal relic density fails [Phys. Rev. D 96, 115010 (2017)]
}

Tobias Binder, Torsten Bringmann®, Michael Gustafsson, and Andrzej Hryczuk

(Q) (Received 11 April 2020; published 8 May 2020)

DOI: 10.1103/PhysRevD.101.099901

Here we address two issues in the originally published version of this article. The first concerns a simple typo for the quantity $v_{\mathrm{CMS}}$ introduced just before Eq. (15); this should read $v_{\mathrm{CMS}}=2 \sqrt{1-4 m_{\chi}^{2} / s}$, which is the relative velocity of the two particles in the center-of-mass frame. This is also the meaning in which $v_{\text {CMS }}$ appears, e.g., in Eq. (40).

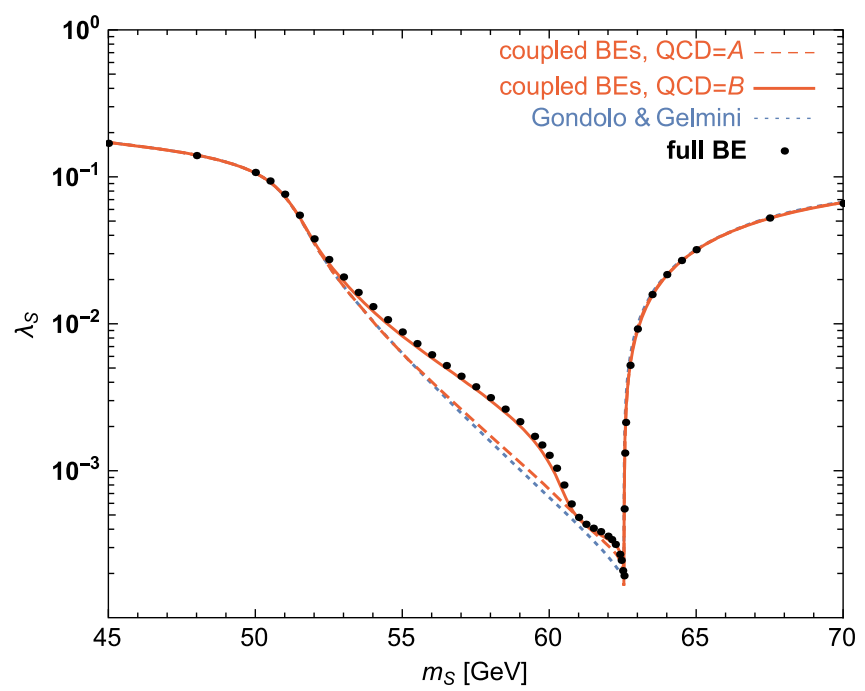

FIG. 1. As Fig. 1 in the originally published version, after replacing Eq. (43) with Eq. (1) in this erratum.

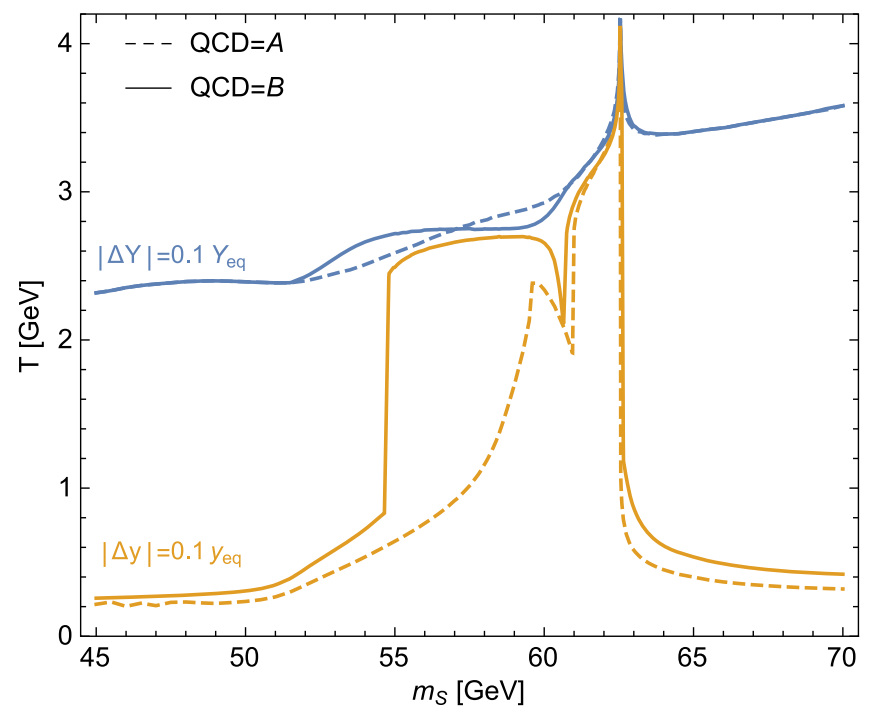

FIG. 2. As Fig. 2 in the originally published version, after replacing Eq. (43) with Eq. (1) in this erratum. 


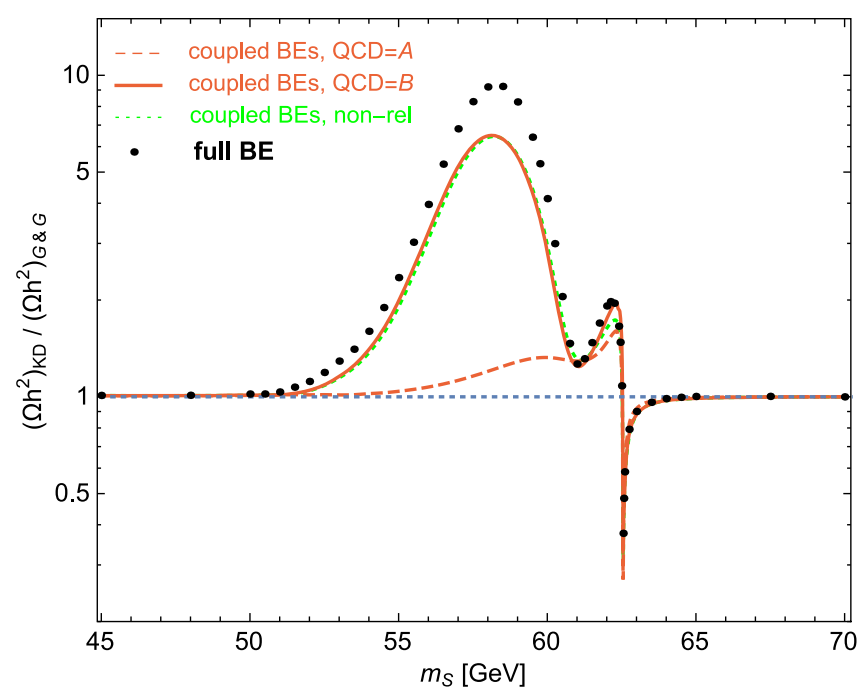

FIG. 3. As Fig. 3 in the originally published version, after replacing Eq. (43) with Eq. (1) in this erratum.
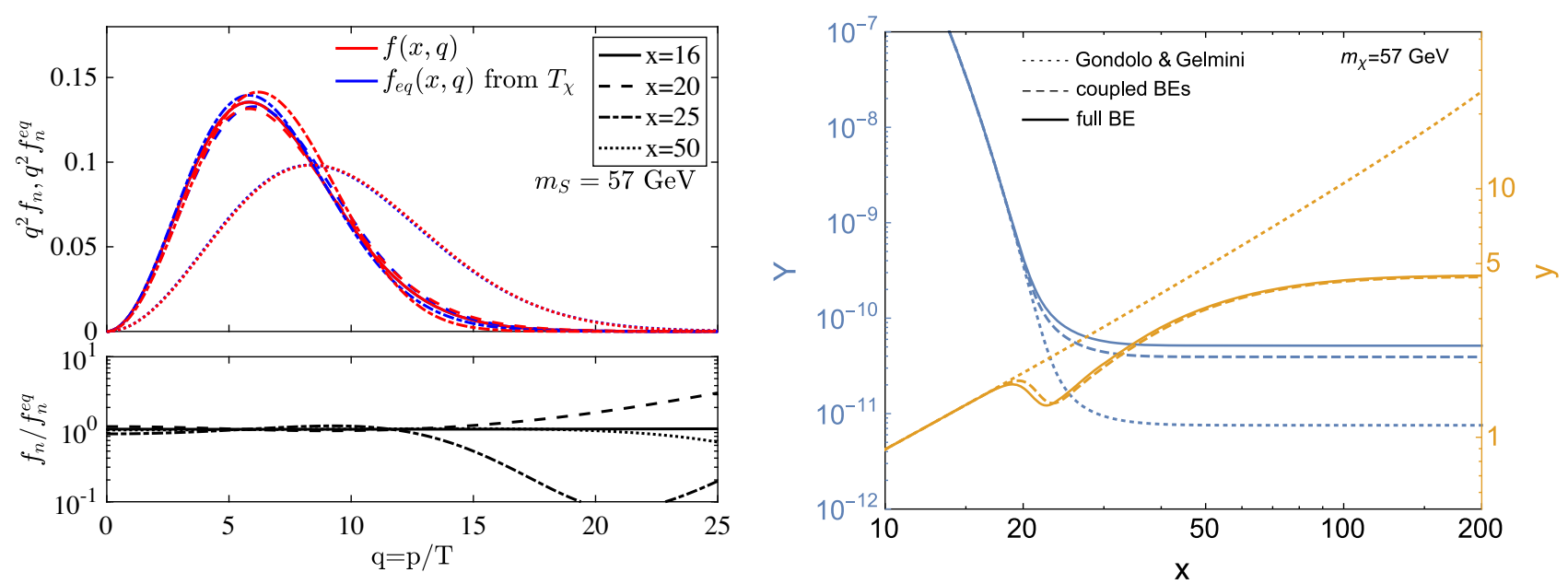

FIG. 4. As Fig. 4 in the originally published version, after replacing Eq. (43) with Eq. (1) in this erratum.

The second point concerns an error in the expressions $(42,43)$ for the elastic scattering rate of scalar singlet dark matter (DM) with standard model (SM) fermions: unlike what is implied in the text, we stated here the spin-averaged result for $|\mathcal{M}|^{2}{ }^{1}$. These expressions should thus be multiplied by a factor of 4 before calculating the momentum exchange rate $\gamma(T)$, as given in Eq. (6), which we did not do in our implementation. For reference, the correct expression for the squared amplitude, after summing over all spin states and averaging over the transferred momentum, is given by

$$
\left\langle|\mathcal{M}|^{2}\right\rangle_{t}=\sum_{f} \frac{N_{f} \lambda_{S}^{2} m_{f}^{2}}{2 k^{4}}\left[\frac{2 k_{\mathrm{cm}}^{2}-2 m_{f}^{2}+m_{h}^{2}}{1+m_{h}^{2} /\left(4 k_{\mathrm{cm}}^{2}\right)}-\left(m_{h}^{2}-2 m_{f}^{2}\right) \log \left(1+4 k_{\mathrm{cm}}^{2} / m_{h}^{2}\right)\right],
$$

where the sum runs over all relevant fermions and antifermions separately.

\footnotetext{
${ }^{1}$ We are grateful to Tomohiro Abe for making us aware of this.
} 

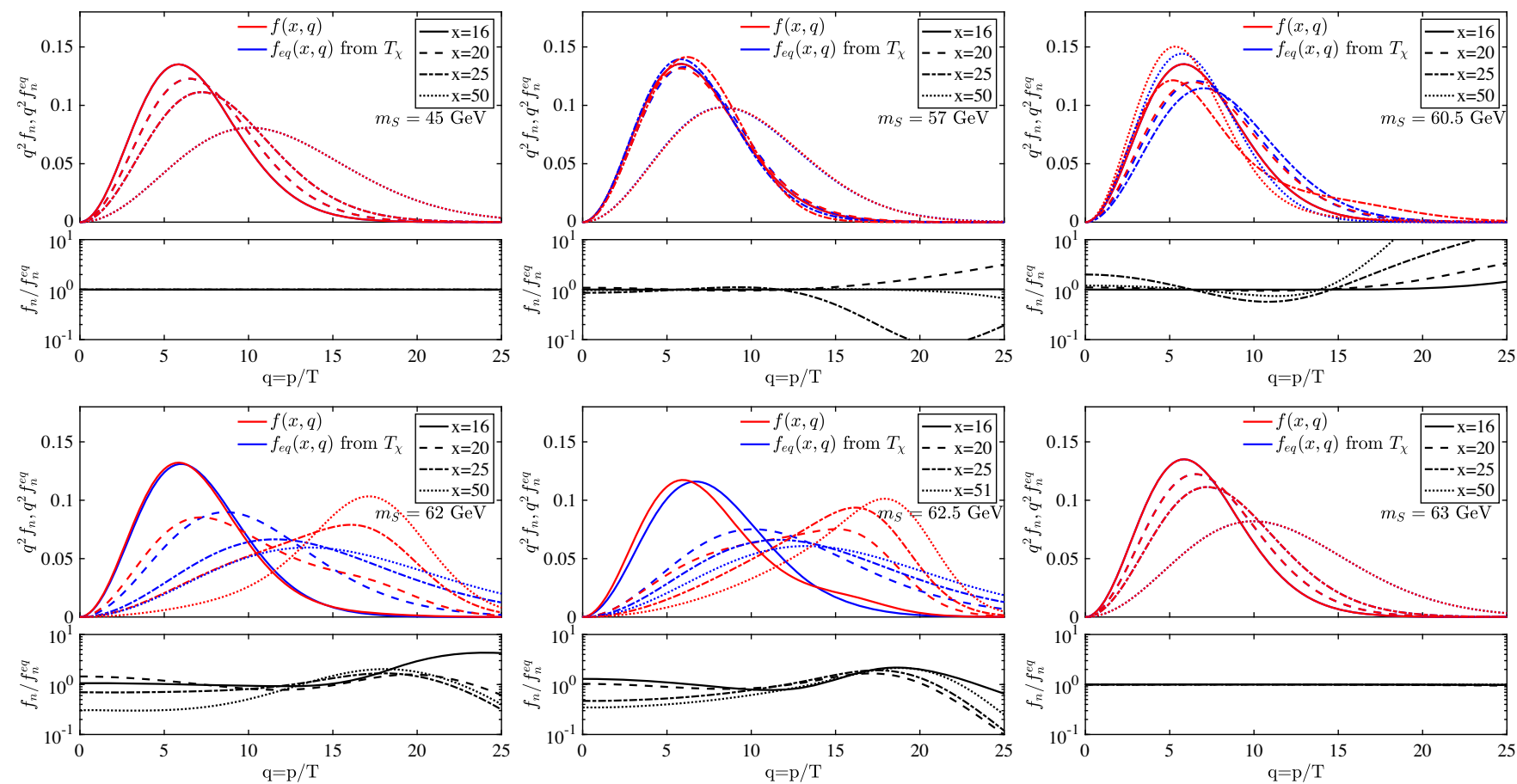

FIG. 5. As Fig. 5 in the originally published version, after replacing Eq. (43) with Eq. (1) in this erratum.
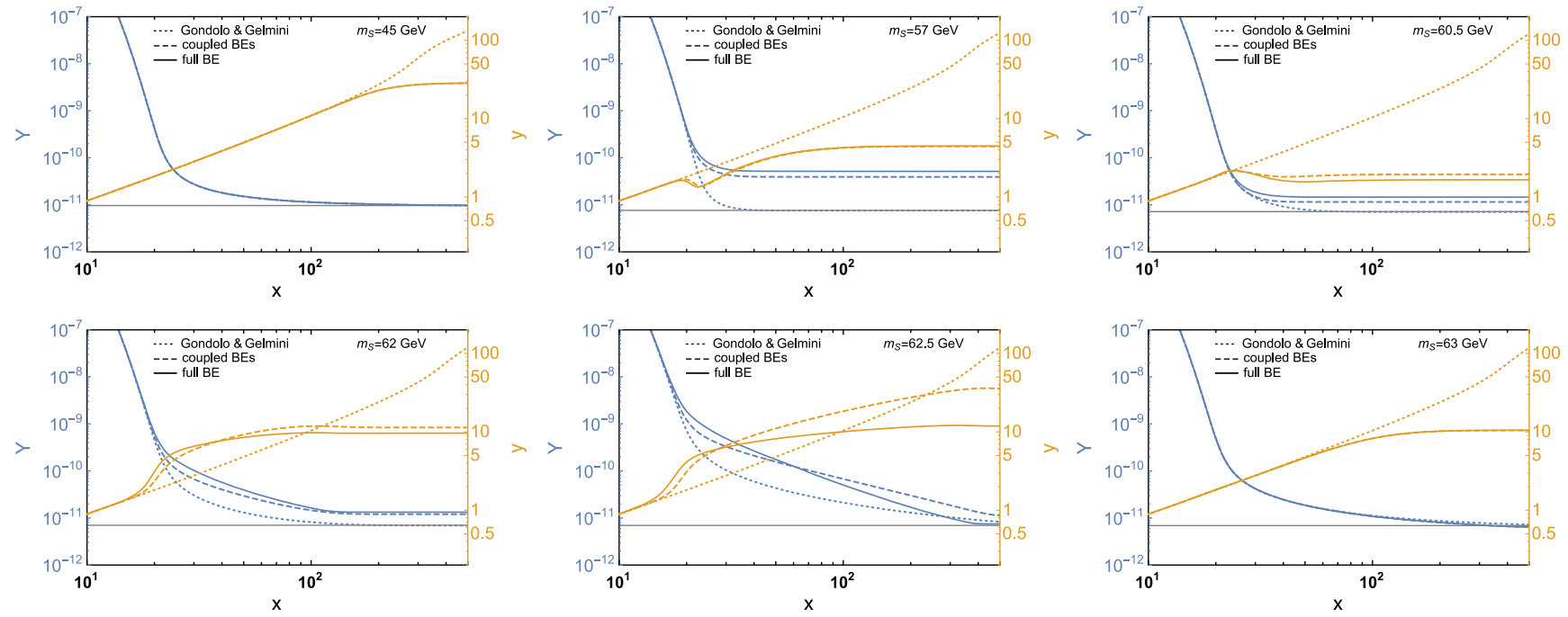

FIG. 6. As Fig. 6 in the originally published version, after replacing Eq. (43) with Eq. (1) in this erratum.

This error does not affect our general discussion (Sec. II) and conclusions, nor does it qualitatively affect the application of our formalism to the scalar singlet model (Sec. III). Quantitatively, however, the larger scattering rate means that the difference to the standard treatment is slightly smaller than what we found originally. This is visible, sometimes only marginally, in every figure of our article. Here we thus present new versions of these figures. Since none of these figures changed qualitatively, and the labels stayed the same, we refer to the originally published version of this article for a detailed description of these figures. 